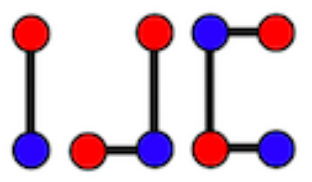

\title{
The total disjoint irregularity strength of some certain graphs
}

\author{
Meilin I. Tilukay ${ }^{\mathrm{a}}$, A.N.M. Salman ${ }^{\mathrm{b}}$ \\ ${ }^{a}$ Department of Mathematics, Faculty of Mathematics and Natural Sciences, \\ Universitas Pattimura, Ambon, Indonesia, \\ ${ }^{b}$ Combinatorial Mathematics Research Group, Faculty of Mathematics and Natural Sciences, \\ Institut Teknologi Bandung, Bandung, Indonesia \\ meilin.tilukay@fmipa.unpatti.ac.id,msalman@math.itb.ac.id
}

\begin{abstract}
Under a totally irregular total $k$-labeling of a graph $G=(V, E)$, we found that for some certain graphs, the edge-weight set $W(E)$ and the vertex-weight set $W(V)$ of $G$ which are induced by $k=t s(G), W(E) \cap W(V)$ is a nonempty set. For which $k$, a graph $G$ has a totally irregular total labeling if $W(E) \cap W(V)=\emptyset$ ? We introduce the total disjoint irregularity strength, denoted by $d s(G)$, as the minimum value $k$ where this condition satisfied. We provide the lower bound of $d s(G)$ and determine the total disjoint irregularity strength of cycles, paths, stars, and complete graphs.
\end{abstract}

Keywords: total disjoint irregularity strength, total irregularity strength, irregular total labeling

Mathematics Subject Classification : 05C78

DOI: $10.19184 /$ ijc.2020.4.2.2

\section{Introduction}

Let $G$ be a finite, simple, and undirected graph with the vertex set $V$ and the edge set $E$. Let $f: V \cup E \rightarrow\{1,2, \cdots, k\}$ be a total $k$-labeling. Under $f$, the weight of a vertex $v \in V$ is $w(v)=f(v)+\sum_{u v \in E} f(u v)$ and the weight of an edge $u v \in E$ is $w(u v)=f(u)+f(u v)+f(v)$. If all the vertex (or edge)-weights are distinct then $f$ is called a vertex (or edge) irregular total

Received: 11 April 2020, Revised: 13 December 2020, Accepted: 17 December 2020. 


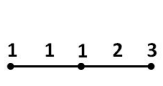

(a)

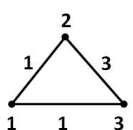

(b)

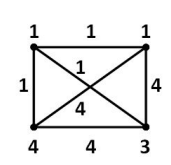

(c)

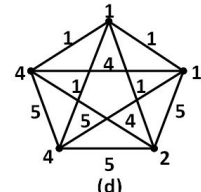

(d)

Figure 1. Totally disjoint irregular total labeling of (a) $P_{3}$, (b) $C_{3}$, (c) $K_{4}$, and (d) $K_{5}$

$k$-labeling and the minimum value $k$ such that $G$ has a vertex (or edge) irregular total $k$-labeling is called the total vertex (or edge) irregularity strength, denoted by $\operatorname{tvs}(G)$ (or tes $(G)$ ), respectively. This parameters were introduced by Baca et al. [2]. They gave the boundary of tes $(G)$ and $\operatorname{tvs}(G)$ and determined that for $n$ vertices, $\operatorname{tvs}\left(C_{n}\right)=\operatorname{tes}\left(C_{n}\right)=\left\lceil\frac{n+2}{3}\right\rceil$, tes $\left(P_{n}\right)=\left\lceil\frac{n+1}{3}\right\rceil$, $\operatorname{tvs}\left(S_{n}\right)=\operatorname{tes}\left(S_{n}\right)=\left\lceil\frac{n+1}{2}\right\rceil$, and $\operatorname{tvs}\left(K_{n}\right)=2$.

Later, Jendrol et al. [7] provided a better lower bound of tes $(G)$ and determined that tes $\left(K_{5}\right)=$ 5 and $\operatorname{tes}\left(K_{n}\right)=\left\lceil\frac{n^{2}-n+4}{6}\right]$, for $n \neq 5$. For any tree $T$, Ivanco and Jendrol [6] proved that $t e s(T)$ is equal to the lower bound. Nurdin et al. [9] gave the lower bound for tvs for any graph $G$.

Recently, Marzuki et al. [8] combined the properties of tes $(G)$ and $\operatorname{tvs}(G)$ and gave new parameter called the total irregularity strength, denoted by $t s(G)$. It is the minimum value $k$ for which $G$ has a totally irregular total $k$-labeling. They proved that the lower bound $t s(G) \geq$ $\max \{\operatorname{tes}(G), \operatorname{tvs}(G)\}$ is sharp for $C_{n}$ and $P_{n}$ except for $P_{2}$ or $P_{5}$. In [14], we proved that for $n \neq 2, t s\left(K_{n}\right)=$ tes $\left(K_{n}\right)$. In [5], Indriati et al. proved that for $n \geq 3, t s\left(S_{n}\right)=t v s\left(S_{n}\right)$. For further reading, one can see [1], [3], [4], [5], and [10] - [13]. All these results showed that the lower bound is sharp.

Observing $t s(G)$, for the vertex weight-set $W(V)$ and the edge weight-set $W(E)$ under a totally irregular total labeling on $G, W(V) \cap W(E) \neq \emptyset$. Considering this condition, we define a new parameter called the total disjoint irregularity strength. A totally disjoint irregular total $k$-labeling of a graph $G$ as a total labeling $f: V \cup E \rightarrow\{1,2, \cdots, k\}$ which satisfies: (i) for any two vertices $x \neq y \in V, w(x) \neq w(y)$; (ii) for any two edges $x_{1} y_{1} \neq x_{2} y_{2} \in E, w\left(x_{1} y_{1}\right) \neq w\left(x_{2} y_{2}\right)$; (iii) $W(V) \cap W(E)=\emptyset$; where $W(V)$ (and $W(E))$ is the vertex (and edge) weight- set, respectively. The minimum value $k$ such that a graph $G$ has a totally disjoint irregular total labeling is called the total disjoint irregularity strength of a graph $G$, denoted by $d s(G)$. Thus, for any graph $G$,

$$
d s(G) \geq t s(G)
$$

For instance, Fig. 1 shows a totally disjoint irregular total labeling of $P_{3}, C_{3}, K_{4}$, and $K_{5}$.

In this paper, we determine $d s$ of cycles, paths, stars, and complete graphs.

\section{Main Results}

Let $G=(V, E)$ be a connected graph. For $G$ has a totally irregular total $k$-labeling $f: V \cup E \rightarrow$ $\{1,2, \cdots, k\}$, we need $|V|+|E|$ distinct weights. Let $\delta=\delta(G)$ (or $\Delta=\Delta(G)$ ) be the minimum (or maximum) degree of vertex in $G$, respectively. Let $n_{i}$ be the number of vertices of degree $i$, where $i=\delta, \delta+1, \cdots, \Delta$. Then $|V|=\sum_{i=\delta}^{\Delta} n_{i}$. Now, assume that $\delta=1$. Let $f$ be a optimal 
labeling with respect to $d s(G)$. Then the maximum weight has to be at least $|E|+|V|+1$. The maximum vertex weight is the sum of $\Delta+1$ labels and every edge weight is the sum of three labels imply that $k \geq\left[\frac{|E|+|V|+1}{\Delta+1}\right]$. Moreover, when $n_{1} \leq \Delta,|V|+|E|$ distinct weights are only exist if $\left\lceil\frac{|E|+|V|+1}{\Delta+1}\right\rceil \geq\left\lceil\frac{|E|+n_{1}+n_{2}+1}{3}\right\rceil$. In the other hand, when $n_{1} \geq \Delta$, we have $2 n_{1}$ distinct weights depend on 2 labels, such that $|V|+|E|$ distinct weights are only exist if $\left\lceil\frac{|E|+|V|+1}{\Delta+1}\right\rceil \geq n_{1}$. Hence, the minimum value $k \geq \max \left\{n_{1},\left\lceil\frac{|E|+n_{1}+n_{2}+1}{3}\right\rceil\right\}$. Next, assume that $\delta \neq 1$. For $f$ is optimal then the minimum weight is at least 3 . Then, $k \geq\left\lceil\frac{|E|+n_{1}+n_{2}+2}{3}\right\rceil$. Thus we have the lower bound of $d s(G)$.

Theorem 2.1. Let $G=(V, E)$ be a connected graph. Let $v$ be a pendant vertex and $n_{i}(i=1,2)$ be the number of vertices of degree $i$. Then

$$
d s(G) \geq \begin{cases}\max \left\{n_{1},\left\lceil\frac{|E|+n_{1}+n_{2}+1}{3}\right\rceil\right\}, & \text { if } v \in V \\ \left\lceil\frac{|E|+n_{1}+n_{2}+2}{3}\right\rceil, & \text { if } v \notin V .\end{cases}
$$

Our next results show that this lower bound is sharp.

Theorem 2.2. Let $m_{1} \geq 3$ and $m_{2} \in \mathbb{N}$. Let $C_{m_{1}}$ be a cycle with $m_{1}$ vertices and $P_{m_{2}}$ be a path with $m_{2}$ vertices. Then

$$
\begin{array}{ll}
d s\left(C_{m_{1}}\right)=\left\lceil\frac{2 m_{1}+2}{3}\right\rceil ; & \\
d s\left(P_{m_{2}}\right)= \begin{cases}3, & \text { for } m_{2}=3 ; \\
\left\lceil\frac{2 m_{2}}{3}\right\rceil, & \text { otherwise. }\end{cases}
\end{array}
$$

To prove Theorem 2.2, we need this lemma.

Lemma 2.1. For any integers $y$ and $x_{i}, 1 \leq i \leq 2 n$, let $\left\{x_{i}\right\}$ be a sequence. If the sum of 3 consecutive integers in $\left\{x_{i}\right\}$ is

$$
x_{i}+x_{i+1}+x_{i+2}= \begin{cases}y+2 i-2, & \text { for } 1 \leq i \leq n-1 \\ y+4 n-2 i-3, & \text { for } n \leq i \leq 2 n-2\end{cases}
$$

then

$$
x_{i}= \begin{cases}x_{2 n-1}, & \text { for } i=n-3 j \text { and } 1 \leq j \leq\left\lceil\frac{2 n}{3}\right\rceil \\ x_{2 n-i+2}, & \text { for } i=n-3 j+1 \text { and } 1 \leq j \leq\left\lceil\frac{2 n}{3}\right\rceil .\end{cases}
$$

Proof. Set all equations above as a linear system leads to the solution which is required.

Now, we are able to prove Theorem 2.2. 
Proof. Let $C_{m_{1}}=\left\{v_{1}, e_{1}, v_{2}, e_{2}, \cdots, v_{m_{1}}, e_{m_{1}}\right\}$ and $P_{m_{2}}=\left\{v_{1}, e_{1}, v_{2}, e_{2}, \cdots, v_{m_{2}}\right\}$. Let $t\left(m_{1}\right)=$ $\left\lceil\frac{2 m_{1}+2}{3}\right\rceil$ and $t\left(m_{2}\right)=\left\lceil\frac{2 m_{2}}{3}\right\rceil$. We divide the proof into two cases as follows:

Case 1. $m_{2}=3$

Suppose that $d s\left(P_{3}\right)=2$. Since we need 5 distinct weight from 2 to 6 , one endpoint (and its incidence edge) can be labeled by 1 to have smallest weight. In the other hand, maximum weight 6 only can occur when the rest vertices and edge are labeled by 2 . Hence, there are one vertex and one edge of the same weight. Contrary to hypothesis. Thus, $d s\left(P_{3}\right) \geq 3$. By label $P_{3}$ as in Fig.1, we have $d s\left(P_{3}\right)=3$.

Case 2. $m_{1} \geq 3$ and $m_{2} \neq 3$

It is trivial for $n_{2}=1$. For $n_{1} \geq 3$ and $n_{2} \geq 2$, by Theorem 2.1, $d s\left(C_{m_{1}}\right) \geq t\left(m_{1}\right)$ and $d s\left(P_{m_{2}}\right) \geq$ $t\left(m_{2}\right)$. For the reverse inequality, we construct $f_{i}: V \cup E \rightarrow\left\{1,2, \cdots, t\left(m_{i}\right)\right\}$, for $i=1$, 2 , as follows: Let $f_{1}^{m_{1}}=\left\{v_{1}^{m_{1}}, e_{1}^{m_{1}}, v_{2}^{m_{1}}, e_{2}^{m_{1}} \cdots v_{m_{1}}^{m_{1}}, e_{m_{1}}^{m_{1}}\right\}$ and $f_{2}^{m_{2}}=\left\{v_{1}^{m_{2}}, e_{1}^{m_{2}}, v_{2}^{m_{2}}, e_{2}^{m_{2}} \cdots v_{m_{2}}^{m_{2}}\right\}$ be the alternating vertex (and edge) label-sets, where $f_{1}\left(v_{i}\right)=v_{i}^{m_{1}}, f_{2}\left(v_{i}\right)=v_{i}^{m_{2}}, f_{1}\left(e_{i}\right)=e_{i}^{m_{1}}, f_{2}\left(e_{i}\right)=$ $e_{i}^{m_{2}}$, and $W\left(C_{m_{1}}\right)=\left\{w\left(v_{i}\right), w\left(e_{i}\right) \mid 1 \leq i \leq m_{1}\right\}$ and $W\left(P_{m_{2}}\right)=\left\{w\left(v_{1}\right), w\left(e_{1}\right), w\left(v_{2}\right), w\left(e_{2}\right), \cdots\right.$, $\left.w\left(v_{m_{2}}\right)\right\}$ be the alternating vertex (and edge) weight-sets of $C_{m_{1}}$ and $P_{m_{2}}$, respectively. Let

$$
d\left(m_{i}\right)= \begin{cases}t\left(m_{i}\right)-1, & \text { for } m_{1} \equiv j \bmod 3, j=0,1, m_{2} \equiv j \bmod 3, j=0,2 ; \\ t\left(m_{i}\right), & \text { for } m_{1} \equiv 2 \bmod 3, m_{2} \equiv 1 \bmod 3 .\end{cases}
$$

We prove by induction on $m_{i}$. For the base step, it is true that for $f_{1}^{3}=\{1,1,2,3,3,1\}, f_{1}^{4}=$ $\{1,2,2,3,3,4,4,1\}$, and $f_{1}^{5}=\{1,1,2,2,3,3,4,4,4,1\}$, we have $W\left(C_{3}\right)=\{3,4,6,8,7,5\}$, $W\left(C_{4}\right)=\{4,5,7,8,10,11,9,6\}$, and $W\left(C_{5}\right)=\{3,4,5,7,8,10,11,12,9,6\}$ and for $f_{2}^{2}=\{1,1,2\}$, $f_{2}^{4}=\{1,1,1,2,2,3,3\}$, and $f_{2}^{6}=\{1,1,1,3,4,4,4,2,3,1,3\}$, we have $W\left(P_{2}\right)=\{2,4,3\}$, $W\left(P_{4}\right)=\{2,3,4,5,7,8,6\}$, and $W\left(P_{6}\right)=\{2,3,5,8,11,12,10,9,6,7,4\}$ such that for $i=1,2$, $f_{i}$ is a totally disjoint irregular total $t\left(m_{i}\right)$ - labeling, $d s\left(C_{m_{1}}\right)=t\left(m_{1}\right)$ for $m_{1} \in\{3,4,5\}$ and $d s\left(P_{m_{2}}\right)=t\left(m_{2}\right)$ for $m_{2} \in\{2,4,6\}$, where the maximum weight is $w\left(e_{d\left(m_{i}\right)}\right)$.

For the inductive step, we assume that for all $k_{1}$ and $k_{2}, f_{1}$ is a totally disjoint irregular total $t_{1}$-labeling of $C_{k_{1}}$ and $f_{2}$ is a totally disjoint irregular total $t_{2}$-labeling of $P_{k_{2}}$, where

$$
\begin{aligned}
e_{d\left(k_{i}\right)}^{k_{i}} & = \begin{cases}t_{i}-1, & \text { for } i=2, k_{2} \equiv j \bmod 9, j \in\{1,2,8\} ; \\
t_{i}, & \text { for } i \in\{1,2\}, k_{2} \equiv j \bmod 9, j \in\{0,3,4,5,6,7\} ;\end{cases} \\
v_{d\left(k_{i}\right)+1}^{k_{i}} & = \begin{cases}t_{i}-1, & \text { for } i \in\{1,2\}, k_{1}=6, k_{2} \equiv j \bmod 9, j \in\{5,7,8\} ; \\
t_{i}, & \text { for } i \in\{1,2\}, k_{1} \neq 6, k_{2} \equiv j \bmod 9, j \in\{0,1,2,3,4,6\} ;\end{cases}
\end{aligned}
$$

and the maximum weight is $w\left(e_{d\left(k_{i}\right)}\right)$.

Let $G_{k_{1}} \cong C_{k_{1}}$ and $G_{k_{2}} \cong P_{k_{2}}$. To prove that $d s\left(G_{\left(k_{i}\right)+3}\right)=t\left(k_{i}+3\right)=d s\left(G_{k_{i}}\right)+2$, we construct $G_{\left(k_{i}\right)+3}$ from $G_{k_{i}}$ by subdividing $e_{d\left(k_{i}\right)}$ as described in Fig.2. Define $f_{i}^{\left(k_{i}\right)+3} \backslash\left\{v_{\left(k_{i}\right)+1}^{\left(k_{i}\right)+3}, e_{\left(k_{i}\right)+1}^{\left(k_{i}\right)+3}, v_{\left(k_{i}\right)+2}^{\left(k_{i}\right)+3}\right.$, $\left.e_{\left(k_{i}\right)+2}^{\left(k_{i}\right)+3}, v_{\left(k_{i}\right)+3}^{\left(k_{i}\right)+3}, e_{\left(k_{i}\right)+1}^{\left(k_{i}\right)+3}\right\}=f_{i}^{k_{i}}$. Setting $w\left(e_{d\left(k_{i}\right)}\right)=w\left(e_{d\left(k_{i}+3\right)-2}\right)$ and $w\left(v_{d\left(k_{i}+3\right)+1}\right)=w\left(v_{d\left(k_{i}\right)+1}\right)$, we have $a \neq b$ for $a, b \in W\left(G_{\left(k_{i}\right)+3}\right) \backslash\left\{v_{\left(k_{i}\right)+1}, e_{\left(k_{i}\right)+1}, v_{\left(k_{i}\right)+2}, e_{\left(k_{i}\right)+2}, v_{\left(k_{i}\right)+3}, e_{\left(k_{i}\right)+1}\right\}$. Moreover, $e_{d\left(k_{i}\right)}^{\left(k_{i}\right)+3}=e_{\left(k_{i}\right)+3}^{\left(k_{i}\right)+3}=e_{d\left(k_{i}\right)}^{\left(k_{i}\right)}$ and $v_{d\left(k_{i}\right)+1}^{\left(k_{i}\right)+3}=v_{\left(k_{i}\right)+1}^{\left(k_{i}\right)+3}=v_{\left.d_{(} k_{i}\right)+1}^{\left(k_{1}\right)}$. This is sufficient to apply Lemma 2.1. Let $\left\{x_{i} \mid 1 \leq i \leq 8\right\}=\left\{e_{d\left(k_{i}\right)}^{\left(k_{i}\right)+3}, v_{\left(k_{i}\right)+1}^{\left(k_{i}\right)+3}, e_{\left(k_{i}\right)+1}^{\left(k_{i}\right)+3}, v_{\left(k_{i}\right)+2}^{\left(k_{i}\right)+3}, e_{\left(k_{i}\right)+2}^{\left(k_{i}\right)+3}, v_{\left(k_{i}\right)+3}^{\left(k_{i}\right)+3}, e_{\left(k_{i}\right)+3}^{\left(k_{i}\right)+3}, v_{d\left(k_{i}\right)+1}^{\left(k_{i}\right)+3}\right\}$ and $y=$ $w\left(e_{d\left(k_{i}\right)}\right)+1$. Then, we have $v_{\left(k_{i}\right)+2}^{\left(k_{i}\right)+3}=e_{d\left(k_{i}\right)}^{\left(k_{i}\right)}+2, e_{\left(k_{i}\right)+2}^{\left(k_{i}\right)+3}=v_{d\left(k_{i}\right)+1}^{\left(k_{i}\right)}+2$, and $v_{\left(k_{i}\right)+3}^{\left(k_{i}\right)+3}-1=e_{\left(k_{i}\right)+1}^{\left(k_{i}\right)+3}$, 


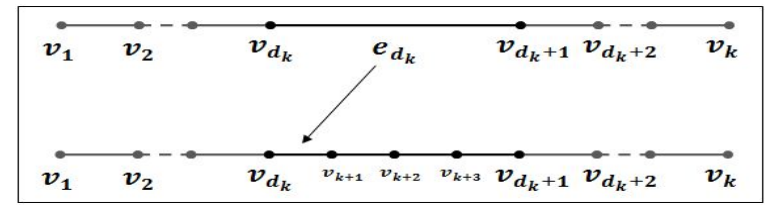

Figure 2. The construction of $P_{k+3}$ from $P_{k}$

where

$$
e_{\left(k_{i}\right)+1}^{\left(k_{i}\right)+3}= \begin{cases}3, & \text { for } i=1, k_{i}=3 \\ t\left(k_{1}\right)+1, & \text { for } i=1, k_{i} \neq 3 \\ 2 k_{i}-2 t\left(k_{i}\right)+3, & \text { for } i=2, k_{2} \equiv 8 \bmod 9 \\ 2 k_{i}-2 t\left(k_{i}\right)+2, & \text { for } i=2, k_{2} \equiv j \bmod 9, j \in\{1,2,5,7\} \\ 2 k_{i}-2 t\left(k_{i}\right)+1, & \text { for } i=2, k_{2} \equiv j \bmod 9, j \in\{0,3,4,6\} .\end{cases}
$$

Then, it can be checked that the maximum label is $d s\left(G_{k_{i}}\right)+2=d s\left(G_{\left(k_{i}\right)+3}\right)$. We have completed the labeling $f_{i}$ on $G_{\left(k_{i}\right)+3}$ and have proved that $f_{i}$ is a totally disjoint irregular total $t\left(k_{i}\right)$-labeling. Thus, for any positive integer $m_{1} \geq 3$ and $m_{2} \in \mathbb{N}, d s\left(C_{m_{1}}\right)=\left\lceil\frac{2 m_{1}+2}{3}\right\rceil, d s\left(P_{m_{2}}\right)=\left\lceil\frac{2 m_{2}}{3}\right\rceil$, for $m_{2} \neq 3$ and $d s\left(P_{3}\right)=3$.

Theorem 2.3. Let $n \in \mathbb{N}, n \geq 3$ and $S_{n}$ be a star with $n+1$ vertices, then $d s\left(S_{n}\right)=n$.

Proof. Let $V\left(S_{n}\right)=\left\{v_{i} \mid 1 \leq i \leq n+1\right\}$ where $v_{n+1}$ is the vertex of degree $n$. By Theorem 2.1, $d s\left(S_{n}\right) \geq n$. To prove the reverse inequality, we construct an irregular total labeling $f: V \cup E \rightarrow$ $\{1,2, \cdots, t\}$ by define $f\left(v_{i}\right)=i$ for $1 \leq i \leq n-1, f\left(v_{n}\right)=n-2, f\left(v_{n+1}\right)=n, f\left(v_{i} v_{n+1}\right)=1$ for $1 \leq i \leq n-1$, and $f\left(v_{n} v_{n+1}\right)=3$. Hence, we have $w\left(v_{i}\right)=i+1$ for $1 \leq i \leq n-1, w\left(v_{n}\right)=n+1$, $w\left(v_{n+1}\right)=2 n+2, w\left(v_{i} v_{n+1}\right)=n+i+1$ for $1 \leq i \leq n-1$, and $w\left(v_{n} v_{n+1}\right)=2 n+1$. See that $W(V) \cap W(E)=\emptyset$. Thus, $f$ is a totally disjoint irregular total labeling and $d s\left(S_{n}\right)=n$ for $n \geq 3$.

Next, by using our previous result in [14], we determine the exact value of $d s\left(K_{n}\right)$. For the convenient of reader, we provided the construction of totally irregular total labeling of $K_{n}$ for $n \neq 5,10,12$ given in [14]. Let $\left[\frac{n^{2}-n+4}{6}\right]=t$ and $\left\lfloor\frac{n+1}{3}\right\rfloor=s$. We divide the vertex-set into 3 mutually disjoint subsets, say $A, B$, and $C$, where $A=\left\{a_{i} \mid 1 \leq i \leq s\right\}, B=\left\{b_{i} \mid 1 \leq i \leq n-2 s\right\}$, and $C=\left\{c_{i} \mid 1 \leq i \leq s\right\}$. Let $f: V \cup E \rightarrow\{1,2, \cdots, t\}$ defined by:

$$
\begin{aligned}
& f\left(a_{i}\right)=1, \quad \text { for } 1 \leq i \leq s ; \\
& f\left(b_{i}\right)=\left(\begin{array}{c}
s \\
2
\end{array}\right)+s(i-1)+1, \text { for } 1 \leq i \leq n-2 s ; \\
& f\left(c_{i}\right)=t, \quad \text { for } 1 \leq i \leq s ; \\
& f\left(a_{i} a_{j}\right)=\left(\begin{array}{c}
j-1 \\
2
\end{array}\right)+i, \quad \text { for } 1 \leq i<j \leq s ; \\
& f\left(a_{i} b_{j}\right)=i, \quad \text { for } 1 \leq i \leq s, 1 \leq j \leq n-2 s ; \\
& f\left(a_{i} c_{j}\right)=s(i-1)+j, \quad \text { for } 1 \leq i, j \leq s ; \\
& f\left(b_{i} b_{j}\right)=s(n-s-i-j+2)-\left(\begin{array}{c}
s \\
2
\end{array}\right)+\left(\begin{array}{c}
j-1 \\
2
\end{array}\right)+i, \text { for } 1 \leq i<j \leq n-2 s ; \\
& f\left(b_{i} c_{j}\right)=\left(\begin{array}{c}
n-2 s \\
2
\end{array}\right)+s(n-s)-t+j+1, \quad \text { for } 1 \leq i \leq n-2 s, 1 \leq j \leq s ; \\
& f\left(c_{i} c_{j}\right)=\left(\begin{array}{c}
n \\
2
\end{array}\right)-2(t-1)-\left(\begin{array}{c}
s-i+1 \\
2
\end{array}\right)+j-i, \text { for } 1 \leq i<j \leq s .
\end{aligned}
$$


Theorem 2.4. Let $n \in \mathbb{N}, n \notin\{i \mid 6 \leq i \leq 59\} \cup\{61,62,65,68,71,74\}$ and $K_{n}$ be a complete graph with $n$ vertices. Then

$$
d s\left(K_{n}\right)= \begin{cases}n, & \text { for } n \leq 5 ; \\ \left\lceil\frac{n^{2}-n+4}{6}\right\rceil, & \text { otherwise. }\end{cases}
$$

Proof. By 1 and Theorem 2.1, $d s\left(K_{n}\right) \geq t s\left(K_{n}\right)$. Let $t=\left\lceil\frac{n^{2}-n+4}{6}\right\rceil$. For the reverse inequality, we divide the proof into three cases as follows:

Case 1. $n \leq 5$

It is obvious for $n \leq 3$. Now, suppose that $d s\left(K_{4}\right)=3$. We need 10 distinct weight with minimum weight 3 . We can label 2 vertices and one edge by label 1 . In the other hand, the maximum weight should be 12. Labeling 3 edges and one vertex by label 3 implies that there are 2 edges with the same weight 7 . Contrary to hypothesis. Thus, $d s\left(K_{4}\right) \geq 4$. To prove the upper bound for $n=4$ or 5, we define $f$ as in Fig. 1. Therefore, we have the exact value of $d s\left(K_{n}\right)$ for $n \leq 5$.

Case 2. $n=77$ or $n \geq 80$

Consider that under the totally irregular total $t$ - labeling of $K_{n}$ in (2), the maximum edge weight is $w\left(c_{s-1} c_{s}\right)=\left(\begin{array}{l}n \\ 2\end{array}\right)+2$ and minimum vertex-weight is $w\left(a_{1}\right)=\frac{s\left(s^{2}-1\right)}{6}+n$. It follow $w\left(c_{s-1} c_{s}\right)<$ $w\left(a_{1}\right)$ implies vertex-weight set and edge weight set are disjoint. Thus, $d s\left(K_{n}\right)=t$ for $n=77$ or $n \geq 80$.

Case 3. $n \in\{60,63,64,66,67,69,70,72,73,75,76,78,79\}$

Consider that under the totally irregular total $t$ - labeling of $K_{n}$ provided in (2), we met condition where the minimum vertex-weight $w\left(a_{1}\right)$ is equal to the weight of an edge connecting vertices in $(B, C)$ or $(C, C)$. Then, we modify $f$. Let $E\left(K_{n}\right)=\left\{e_{i} \mid 1 \leq i \leq n(n-1) / 2\right\}$. Let $e_{p} \in E\left(K_{n}\right)$ be an edge where $w\left(a_{1}\right)=w\left(e_{p}\right)$. Since $t \equiv 2 \bmod 3$, then $f\left(e_{n(n-1) / 2}\right)=f\left(c_{s-1} c_{s}\right)=t-1$. It implies that we can change $f\left(e_{i}\right)$ by $f\left(e_{i}+1\right)$, for $p \leq i \leq n(n-1) / 2$ without changing the maximum label such that $W\left(V\left(K_{n}\right)\right) \cap W\left(E\left(K_{n}\right)\right)=\emptyset$. It complete the proof.

\section{Open Problem}

1. For $n \in \mathbb{N}, n \in\{i \mid 6 \leq i \leq 59\} \cup\{61,62,65,68,71,74\}$, find the exact value of $d s\left(K_{n}\right)$.

2. For any graph $G$, find $d s(G)$.

\section{References}

[1] A. Achmad, M. Ibrahim, and M.K. Siddiqui, On the total irregularity strength of generalized Petersen graph, Math. Reports. 18 (68) (2016), 197-204.

[2] M. Bača, S. Jendrol', M. Miller, and J. Ryan, On irregular total labelings, Discrete Math. 307 (2007), 1378-1388.

[3] J.A. Galian, A dynamic survey of graph labeling, Electron. J. Comb. 18 (2015), $\sharp$ DS6.

[4] D. Indriati, Widodo, I.E. Wijayanti, and K.A. Sugeng, On the total irregularity strength of double-star and related graphs, Procedia Comput. Sci. 74 (2015), 118-123. 
[5] D. Indriati, Widodo, I.E. Wijayanti, and K.A. Sugeng, On the total irregularity strength of star graphs, double-stars and caterpillar, AIP Conf. Proc. 1707 (2016), 020008-1-020008-6.

[6] J. Ivančo and S. Jendrol̆, Total edge irregularity strength of trees, Discuss. Math. Graph Theory. 26 (2006), 449-456.

[7] S. Jendro $\check{l}$, J. Miskuf, and R. Sotăk, Total edge irregularity strength of complete graphs and complete bipartite graphs, Discrete Math. 310 (2010), 400-407.

[8] C.C. Marzuki, A.N.M. Salman, and M. Miller, On the total irregularity strengths of cycles and paths, Far East J. Math. Sci. 82(1) (2013), 1-21.

[9] Nurdin, E.T. Baskoro, A.N.M. Salman, and N.N. Gaos, On the total vertex irregularity strength of trees, Discrete Math. 310 (2010), 3043-3048.

[10] R. Ramdani, and A.N.M. Salman, On the total irregularity strength of some cartesian product graphs, AKCE Int. J. Graphs Comb. 10 2013, 199-209.

[11] R. Ramdani, A.N.M. Salman, and H. Assiyatun, On the total irregularity strength of regular graphs, J. Math. Fund. Sci. 47(3) (2015), 281-295.

[12] R. Ramdani, A.N.M. Salman, H. Assiyatun, A. Semaničovă-Feňovčikovă, and M. Bača, Total irregularity strength of three families of graphs, Math. Comput. Sci. 9 (2015), 229-237.

[13] M. Tilukay, A.N.M. Salman, and E.R. Persulessy, On the total irregularity strength of fan, wheel, triangular book, and friendship graphs, Procedia Comput. Sci. 74 (2015), 124-131.

[14] M. Tilukay, B.P. Tomasouw, F.Y. Rumlawang, and A.N.M. Salman, The total irregularity strength of complete graphs and complete bipartite graphs, Far East J. Math. Sci. 102(2) (2017), 317-327. 